\title{
Originales
}

\section{Mejoría en la asistencia al síndrome coronario agudo en los centros de asistencia primaria mediante un plan de formación}

\author{
M. PIQUÉ-GILART'a, J. TORRES-PUIG-GROS ${ }^{b}$, B. BALSERA-GARRIDO ${ }^{c}$, I. TARRUELLA-VIDAL $^{d}$, \\ J. LOSCOS-SOLÉe Y E. PUEO-CRESPOa \\ aServicio de Cardiología. Hospital Universitario Arnau de Vilanova. Lleida. \\ bServicios Territoriales del Departamento de Salud. Lleida. \\ 'Unidad de Cuidados Intensivos. Hospital Universitario Arnau de Vilanova. Lleida. \\ ¿Área Básica de Salud de Tárrega. Lleida. \\ eÁrea Básica de Salud de Agramunt. Lleida.
}

\begin{abstract}
Otros autores corporativos del grupo MIRALL (miocardi isquèmic ràpidament assistit a Lleida): M. Almirall- Egerique, G. Amat-Camats, S. Barranco-Tomàs, M. Maria Bretcha-Vivó, J. Cabré-Ollé, C. Cardona-Cabasés, M. Galán-Seuma, J.M. Guiu-Bardají, J.M. Lara-Nieto, M. Tersa-Alcobé y T. Vilá-Parrot
\end{abstract}

Objetivo. Evaluar preliminarmente el impacto de un programa de formación en la atención prehospitalaria al síndrome coronario agudo (SCA).

Diseño. Estudio transversal.

Ámbito. Área de Salud de Lleida.

Pacientes. Seiscientos sesenta y un casos de SCA atendidos en un Servicio de Medicina Intensiva (SMI) del 1 de enero de 2002 al 31 de diciembre de 2004.

Intervenciones. Se realizó un plan de formación para médicos de asistencia primaria sobre manejo prehospitalario del SCA entre julio de 2003 y diciembre de 2006 en 29 centros de salud.

Principales variables de interés. Se midió la evolución de la prevalencia de aplicación de 5 medidas prehospitalarias (ácido acetilsalicílico, nitroglicerina, electrocardiograma, vía venosa y cloruro mórfico endovenoso) a lo largo del período de estudio.

Resultados. En los centros con plan de formación finalizado se observó un incremento evidente en la aplicación de las 5 medidas prehospitalarias a lo largo del período de estudio. La apertura

Correspondencia: Dr. M. Piqué-Gilart.

C/ Sant Jordi, 4

25123 Torrefarrera. Lleida.

Correo electrónico: mpique@arnau.scs.es

Manuscrito aceptado el 27-IV-2007. de una vía intravenosa, la administración de cloruro mórfico intravenoso y la administración de ácido acetilsalicílico multiplican su aplicación por 10, 8 y 3 respectivamente.

Conclusiones. Un adecuado plan de formación permite mejorar la asistencia de estos pacientes por parte de los médicos de cabecera.

PALABRAS CLAVE: Atención Primaria, cardiopatía isquémica, evaluación de programas.

\section{THE EFFECTIVENESS OF TRAINING SCHEMES IN THE TREATMENT OF ACUTE CORONARY SYNDROME AT HEALTH CENTERS}

Objective. A preliminary evaluation of the impact of a training program on pre-hospitalization care of acute coronary syndrome (ACS).

Design. A cross-sectional study.

Setting. Lleida Health Service area.

Patients. A total of 661 ACS cases were observed in the Intensive Medical Care Unit from January $1^{\text {st }} 2002$ to December $31^{\text {th }} 2004$.

Interventions. A training program for primary health care medical staff on the pre-hospitalization management of ACS from July 2003 to December 2006 in 29 health care centers was conducted.

Main endpoints of interest. The evolution of 5 pre-hospitalization interventions, (acetylsalicylic acid, nitroglycerine, electrocardiogram, intra- 
venous tube and intravenous morphine) throughout the study period was measured.

Results. It was noted during the study that those centers in which training programs were held had a clear increase in the use of the 5 prehospitalization interventions. Insertion of an intravenous line, administration of intravenous morphine and administration of acetylsalicylic acid were all observed to have multiplied their use 10 , 8 and 3 times, respectively.

Conclusions. A training scheme for general practitioners (GP) is essential to ensure appropriate care of patients with this condition.

KEY WORDS: Primary Health Care, ischemic heart disease, evaluation programs.

\section{INTRODUCCIÓN}

La elevada morbimortalidad del síndrome coronario agudo (SCA) ha conllevado en nuestro país el diseño de planes generales de mejora asistencial ${ }^{1,2}$. El espectacular despliegue de las técnicas de revascularización, de marcado o exclusivo carácter hospitalario, no debe hacer olvidar las aportaciones de otras intervenciones más sencillas. El papel de la asistencia primaria en la atención de estos pacientes cabe ser definido como fundamental, no sólo por la necesidad de rápida identificación de la patología, sino también por la capacidad de iniciar medidas contrastadas y recomendadas por las sociedades científicas para el beneficio de estos pacientes.

La administración de ácido acetilsalicílico (AAS) muestra una eficiencia terapéutica difícilmente igualable en el SCA. La marcada reducción de la morbimortalidad la hacen imprescindible en la asistencia de estos pacientes ${ }^{3}$. Su disponibilidad y facilidad de administración han propiciado que la Joint Comission on Accreditation of Healthcare Organizations la tenga incluida como estándar de acreditación ${ }^{4}$.

La nitroglicerina (NTG) sublingual constituye otro estándar terapéutico y, aunque no ha podido demostrar reducciones claras en la mortalidad, puede yugular las crisis con componente vasoespástico ${ }^{5}$.

Obtener un electrocardiograma (ECG) en la fase prehospitalaria posee la capacidad de reducir los tiempos asistenciales e iniciar precozmente la estratificación del riesgo ${ }^{6,7}$.

Establecer una vía venosa permeable y aliviar el dolor con cloruro mórfico aportará confort al paciente $\mathrm{y}$, probablemente, reducirá también los tiempos asistenciales, aunque este punto no ha sido estudiado ${ }^{8,9}$.

Con la finalidad de optimizar la asistencia prehospitalaria al SCA en el Área de Salud de Lleida se elaboró un plan de formación. El presente estudio pretende evaluar preliminarmente dicha intervención. En concreto, medir la evolución de la prevalencia de aplicación de estas medidas prehospitalarias a lo largo del período 2002-2004 en 5 centros de salud (CS) intervenidos al completo durante 2003, y en los restantes $24 \mathrm{CS}$ que no completaron su formación hasta 2006.

\section{PACIENTES Y MÉTODOS}

Desde el segundo semestre de 2003 hasta finales de 2006 se aplicó un plan de formación sobre el manejo prehospitalario del SCA en los 29 centros de salud (CS) que componen nuestra área sanitaria. Se diseñó un curso de 8 horas, dirigido a todos los médicos de cabecera, impartido por especialistas de los Servicios de Medicina Intensiva y Cardiología del Hospital Universitario Arnau de Vilanova, y por varios médicos de asistencia primaria previamente adiestrados. El contenido de dicho curso revisaba las actuaciones en soporte vital y el manejo inicial del SCA, incidiendo en la importancia de las 5 intervenciones prehospitalarias anteriormente citadas. Además, se elaboró un material docente consistente en un cuaderno docente y una guía de bolsillo, que se entregó a todos los médicos de cabecera, y un póster recopilatorio que se repartió por todos los CS. Para llevar a cabo el proyecto se constituyó un grupo de trabajo multidisciplinar que, bajo el acrónimo MIRALL (miocardi isquèmic ràpidament assistit a Lleida), engloba profesionales sanitarios de distintos niveles asistenciales.

Se estableció un calendario para la intervención en todos los CS de una forma gradual durante el período de formación, intentando minimizar la interferencia en la asistencia diaria. Sin embargo, a fin de validar el método, se eligieron 5 centros de salud para ser intervenidos al completo durante el segundo semestre de 2003. El resto de centros completó su formación en 2006 .

Se realizó un estudio descriptivo transversal que incluyó todos los pacientes atendidos por SCA en la Unidad de Medicina Intensiva (UMI) del Hospital Universitario Arnau de Vilanova de Lleida, hospital de referencia para toda la provincia de Lleida, entre el 1 de enero de 2002 y el 31 de diciembre de 2004. En todos ellos se analizó la aplicación, o no, de las 5 medidas básicas de atención prehospitalaria (AAS, NTG, ECG, vía venosa y cloruro mórfico endovenoso). La información se registró mediante la base de datos del Proyecto ARIAM ${ }^{10}$. La medida de frecuencia fue la prevalencia expresada en porcentajes. Las variaciones de prevalencia entre años se analizaron a través de la prueba de Chi-cuadrado; además se analizó la tendencia lineal a lo largo del período de estudio a través de la odds ratio (OR) y el test de tendencia lineal, tomando el primer año del período como OR basal $(\mathrm{OR}=1)$. Los datos se analizaron con los programas Epiinfo v 3.3 y EPIDAT v 3.0. El nivel de significación estadística aceptado fue de $\mathrm{p}<0,05$.

\section{RESULTADOS}

El total de episodios de SCA del conjunto del Área de Salud de Lleida atendidos en la UMI durante el período de estudio fue de 661 , de los que $142(21,5 \%)$ 
PIQUÉ-GILART M ET AL. MEJORÍA EN LA ASISTENCIA AL SÍNDROME CORONARIO AGUDO EN LOS CENTROS DE ASISTENCIA PRIMARIA MEDIANTE UN PLAN DE FORMACIÓN

TABLA 1. Características sociodemográficas y clínicas de los pacientes atendidos por síndrome coronario agudo

\begin{tabular}{|l|c|c|}
\hline & $\begin{array}{c}\text { Pacientes de los 5 CS intervenidos } \\
\text { a final de 2003(n=142) }\end{array}$ & $\begin{array}{c}\text { Pacientes de los restantes 24 CS } \\
(\mathrm{n}=519)\end{array}$ \\
\hline Edad, años & $68,7 \pm 17,3$ & $65,7 \pm 16,3$ \\
\hline Hombres & $111(78 \%)$ & $409(79 \%)$ \\
\hline Diagnóstico al alta: & $109(77 \%)$ & $383(74 \%)$ \\
IAM & $33(23 \%)$ & $136(26 \%)$ \\
Angina & $11(8 \%)$ & $32(6 \%)$ \\
\hline Mortalidad hospitalaria & & $\mathrm{NS}$ \\
\hline Aplicación prehospitalaria antes del plan & & \\
de formación de las siguientes medidas: & $8 / 40(20 \%)$ & $61 / 126(48 \%)$ \\
AAS oral o endovenoso & $32 / 40(80 \%)$ & $91 / 126(72 \%)$ \\
NTG sublingual & $27 / 40(67 \%)$ & $88 / 126(70 \%)$ \\
ECG & $2 / 40(5 \%)$ & $28 / 126(22 \%)$ \\
Vía venosa & $2 / 40(5 \%)$ & $16 / 126(13 \%)$ \\
Cloruro mórfico endovenoso & NS & \\
\hline
\end{tabular}

AAS: ácido acetilsalicílico; CS: centros de salud; ECG: electrocardiograma; IAM: infarto agudo de miocardio; NS: no significativo; NTG: nitroglicerina.

procedían de los 5 CS donde se había realizado la intervención al completo en 2003. Las características sociodemográficas y clínicas de los pacientes se presentan en la tabla 1; en ella destaca la menor utilización de dos de las medidas valoradas, AAS y vía venosa en los 5 CS inicialmente intervenidos respecto del resto de centros.

Los resultados obtenidos en la aplicación de las 5 medidas prehospitalarias durante el período 2002-
2004 en los 5 centros intervenidos completamente se muestran en la tabla 2 . Los resultados preliminares referidos al resto de CS se presentan en la tabla 3 .

En los 5 centros donde se había completado la intervención el incremento en la aplicación de estas 5 medidas fue más evidente excepto para la NTG, ya que en estos centros se partía de un nivel de aplicación más elevado. En ellos, al final del período evaluado, la vía venosa prácticamente llegó a multiplicar

TABLA 2. Evolución de la aplicación prehospitalaria de 5 medidas en el manejo del síndrome coronario agudo durante el período 2002-2004 en los 5 centros de salud intervenidos al completo al final del 2003 en el Área de Salud de Lleida

\begin{tabular}{|c|c|c|c|}
\hline Medida & Período (pacientes) & Prevalencia & Odds ratio (IC 95\%) \\
\hline \multirow[t]{6}{*}{ AAS vía oral o endovenosa } & $2002(\mathrm{n}=40)$ & $8(20 \%)$ & 1 \\
\hline & $2003(\mathrm{n}=60)$ & $19(32 \%)$ & $1,7(0,7-4,7)$ \\
\hline & $2004(n=42)$ & $28(67 \%)$ & $7,5(2,6-18,5)$ \\
\hline & Total $(n=142)$ & $58(41 \%)$ & \\
\hline & $\mathrm{p}$ & 0,003 & \\
\hline & $\mathrm{p}$ tendencia lineal & 0,001 & \\
\hline \multirow[t]{6}{*}{ NTG sublingual } & $2002(n=40)$ & $32(80 \%)$ & 1 \\
\hline & $2003(\mathrm{n}=60)$ & $42(70 \%)$ & $0,7(0,2-1,5)$ \\
\hline & $2004(\mathrm{n}=42)$ & $36(86 \%)$ & $1,5(0,5-4,6)$ \\
\hline & Total $(n=142)$ & $109(77 \%)$ & \\
\hline & p & 0,31 & \\
\hline & $\mathrm{p}$ tendencia lineal & 0,52 & \\
\hline \multirow[t]{6}{*}{ ECG } & $2002(n=40)$ & $27(67 \%)$ & 1 \\
\hline & $2003(\mathrm{n}=60)$ & $41(68 \%)$ & $1,0(0,4-2,4)$ \\
\hline & $2004(\mathrm{n}=42)$ & $42(100 \%)$ & NA \\
\hline & Total $(n=142)$ & $113(78 \%)$ & \\
\hline & p & 0,05 & \\
\hline & $\mathrm{p}$ tendencia lineal & 0,05 & \\
\hline \multirow[t]{6}{*}{ Vía venosa } & $2002(n=40)$ & $2(5 \%)$ & 1 \\
\hline & $2003(n=60)$ & $19(32 \%)$ & $5,0(2,1-40,3)$ \\
\hline & $2004(n=42)$ & $20(48 \%)$ & $16,7(4,1-81,0)$ \\
\hline & Total $(n=142)$ & $38(27 \%)$ & \\
\hline & $\mathrm{p}$ & 0,002 & \\
\hline & $\mathrm{p}$ tendencia lineal & 0,001 & \\
\hline \multirow[t]{6}{*}{ Cloruro mórfico endovenoso } & $2002(n=40)$ & $2(5 \%)$ & 1 \\
\hline & $2003(n=60)$ & $11(18 \%)$ & $4,1(1,0-20,0)$ \\
\hline & $2004(\mathrm{n}=42)$ & $16(38 \%)$ & $10,6(2,7-55,2)$ \\
\hline & Total $(n=142)$ & $31(22 \%)$ & \\
\hline & $\mathrm{p}$ & 0,024 & \\
\hline & $\mathrm{p}$ tendencia lineal & 0,009 & \\
\hline
\end{tabular}

AAS: ácido acetilsalicílico; ECG: electrocardiograma; NA: no aplicable; NTG: nitroglicerina; IC: intervalo de confianza. 
PIQUÉ-GILART M ET AL. MEJORÍA EN LA ASISTENCIA AL SÍNDROME CORONARIO AGUDO EN LOS CENTROS DE ASISTENCIA PRIMARIA MEDIANTE UN PLAN DE FORMACIÓN

TABLA 3. Evolución de la aplicación prehospitalaria de 5 medidas en el manejo del síndrome coronario agudo durante el período 2002-2004 en los 24 centros de salud del Área de Salud de Lleida en los que la intervención se completó en 2006

\begin{tabular}{|c|c|c|c|}
\hline Medida & Período (pacientes) & Prevalencia & Odds ratio (IC 95\%) \\
\hline \multirow[t]{6}{*}{ AAS vía oral o endovenosa } & $2002(n=126)$ & $61(48 \%)$ & 1 \\
\hline & $2003(n=210)$ & $95(45 \%)$ & $0,9(0,6-1,4)$ \\
\hline & $2004(n=183)$ & $107(58 \%)$ & $1,5(0,9-2,4)$ \\
\hline & Total $(n=519)$ & $265(51 \%)$ & \\
\hline & $\mathrm{p}$ & 0,27 & \\
\hline & $\mathrm{p}$ tendencia lineal & 0,49 & \\
\hline \multirow[t]{6}{*}{ NTG sublingual } & $2002(n=126)$ & $91(72 \%)$ & 1 \\
\hline & $2003(n=210)$ & $164(78 \%)$ & $1,4(0,8-2,3)$ \\
\hline & $2004(n=183)$ & $164(90 \%)$ & $3,3(1,8-6,1)$ \\
\hline & Total $(n=519)$ & $424(82 \%)$ & \\
\hline & $\mathrm{p}$ & 0,003 & \\
\hline & $\mathrm{p}$ tendencia lineal & 0,001 & \\
\hline \multirow[t]{6}{*}{ ECG } & $2002(n=126)$ & $88(70 \%)$ & 1 \\
\hline & $2003(n=210)$ & $147(70 \%)$ & $1,0(0,6-1,6)$ \\
\hline & $2004(n=183)$ & $138(75 \%)$ & $1,3(0,8-2,2)$ \\
\hline & Total $(n=519)$ & $373(72 \%)$ & \\
\hline & $\mathrm{p}$ & 0,416 & \\
\hline & $\mathrm{p}$ tendencia lineal & 0,248 & \\
\hline \multirow[t]{6}{*}{ Vía venosa } & $2002(n=126)$ & $28(22 \%)$ & 1 \\
\hline & $2003(n=210)$ & $49(23 \%)$ & $1,1(0,6-1,8)$ \\
\hline & $2004(n=183)$ & $81(44 \%)$ & $2,3(1,7-4,6)$ \\
\hline & Total $(n=519)$ & $166(32 \%)$ & \\
\hline & $\mathrm{p}$ & 0,001 & \\
\hline & $\mathrm{p}$ tendencia lineal & 0,001 & \\
\hline \multirow[t]{6}{*}{ Cloruro mórfico endovenoso } & $2002(n=126)$ & $16(13 \%)$ & 1 \\
\hline & $2003(n=210)$ & $35(17 \%)$ & $1,4(0,7-2,6)$ \\
\hline & $2004(n=183)$ & $54(29 \%)$ & $2,9(1,6-5,3)$ \\
\hline & Total $(n=519)$ & $107(21 \%)$ & \\
\hline & $\mathrm{p}$ & 0,0004 & \\
\hline & $\mathrm{p}$ tendencia lineal & 0,0002 & \\
\hline
\end{tabular}

AAS: ácido acetilsalicílico; ECG: electrocardiograma; NTG: nitroglicerina; IC: intervalo de confianza.

por 10 su nivel de aplicación, la administración intravenosa de cloruro mórfico prácticamente por 8 y la de AAS por 3. En los tres casos se observó un considerable grado de asociación entre la intervención y sus resultados, medida a través de la OR llegando a 16,7 (IC 95\%: 4,1-81,0), 10,6 (IC 95\%: 2,7-55,2) y 7,5 (IC 95\%: 2,6-18,5) respectivamente. Esta asociación fue corroborada por su tendencia lineal. La NTG y el ECG partían de un elevado nivel de aplicación. El incremento más acentuado de la OR se produce entre 2003 y 2004, especialmente en el AAS y el ECG. De todas formas los resultados de la intervención ya se empiezan a percibir en el 2003 en estos 5 centros intervenidos.

En los $24 \mathrm{CS}$ restantes, que se intervinieron posteriormente, se observó un incremento significativo y lineal en la aplicación de las medidas prehospitalarias, excepto para el ECG, que partía de un nivel básico de aplicación elevado (casi tres cuartas partes).

\section{DISCUSIÓN}

La aproximación a la asistencia primaria por parte de la especializada puede producir efectos beneficiosos muy evidentes. Los resultados de nuestro programa docente nos permiten vislumbrar una serie de cuestiones. Por una parte, observamos la necesidad de formación o puesta al día en el manejo de los pacientes con SCA. Por otra parte, hemos podido cons- tatar que la utilización de estos recursos asistenciales en el manejo de este tipo de patologías por parte de los médicos de cabecera es manifiestamente mejorable. Así, fijándonos en las 5 variables monitorizadas, constatamos que únicamente la administración de NTG y la obtención del ECG consiguen, basalmente, sobrepasar el $50 \%$, quedando el resto -incluyendo el eficiente ácido acetilsalicílico- en niveles testimoniales. La uniforme mejoría en todas las variables, rápidamente conseguida en los 5 centros intervenidos inicialmente, nos permite aventurar un manifiesto cambio en el manejo de todos estos pacientes cuando se haya completado el plan de formación, para cuya evaluación deberemos esperar algunos años.

Resulta difícil cuantificar el beneficio clínico conseguido. Sin embargo, el aumento absoluto superior al $20 \%$ en la administración precoz de AAS debería traducirse en una disminución de la morbimortalidad. Mantener, e incluso aumentar, las ya elevadas tasas de administración de NTG y obtención de un ECG, o triplicar la implantación de una vía venosa y administración de cloruro mórfico, ha de redundar en el confort del paciente, la agilización del proceso y la mejoría en los tiempos asistenciales, factores todos ellos que inciden positivamente sobre la evolución clínica.

Nuestro estudio no abarca la totalidad de los pacientes que acudieron al hospital Universitari Arnau de Vilanova de Lleida presentando un SCA, 
lo que constituye una limitación del estudio. No obstante, dado que los criterios de ingreso no han variado durante este tiempo, y que el mayor determinante ha sido la aleatoria disponibilidad de camas en la Unidad de Cuidados Intensivos, consideramos la muestra representativa de la globalidad y sus datos, extrapolables.

Las diferencias iniciales en la aplicación de AAS y vía venosa, entre los dos grupos de centros de salud en estudio, se debe a los criterios de selección de los CS a intervenir prioritariamente. Estos 5 centros se eligieron por concurrir en ellos la pertenencia a un ambiente rural, alejados del hospital y consecuentemente con tiempos de tratamiento más prolongados, y que presentasen bajas tasas de utilización de estas 5 medidas estudiadas. A pesar de la ya mencionada distinta situación de partida, dichos centros consiguieron un importante incremento en la aplicación de estas medidas, especialmente en la administración de AAS.

Son muy escasas las experiencias que evalúan la bondad de un plan de formación en el ámbito de una amplia área geográfica ${ }^{11}$. Nuestro estudio muestra que los planes de formación constituyen un instrumento útil para mejorar la asistencia de los SCA en el medio prehospitalario. El seguimiento en los próximos años nos permitirá completar los resultados una vez finalizado el plan de formación. Sin embargo, y dada la mejoría significativa conseguida, consideramos procedente presentar la situación actual como el inicio de un camino que, probablemente, valga la pena seguir.

\section{Declaración de conflicto de intereses}

Los autores han declarado no tener ningún conflicto de intereses.

\section{BIBLIOGRAFÍA}

1. Plan integral de cardiopatía isquémica. Madrid: Ministerio de Sanidad y Consumo; 2003.

2. PAICI. Pla d'Atenció Integral a la Cardiopatia Isquémica. Generalitat de Catalunya; 2003. Accedido el 28 de octubre de 2006. Disponible en: http://www10.gencat.net/catsalut/archivos/ publicacions.

3. Antiplatelet Trialist Collaboration. Collaborative overview of randomised trials of antiplatelet therapy-I: prevention of death, myocardial infarction, and stroke by prolonged antiplatelet therapy in various categories of patients. BMJ. 1994;308:81-106.

4. Joint Commission on Acreditation of Healthcare Organizations. 1994. Acreditation Manual for Hospitals. Volume I. Standards. Oakbrook Terrace: JCAHO; 1993.

5. Hennekens CH, Albert CM, Godfried SL, Gaziano JM, Buring JE. Adjunctive drug therapy of acute myocardial infarctionevidence from clinical trials. N Engl J Med. 1996;335:1660-7.

6. Canto JG, Rogers WJ, Bowlby LJ, French WJ, Pearce DJ, Weaver WD. The prehospital electrocardiogram in acute myocardial infarction: is its full potential being realized? National Registry of Myocardial Infarction 2 Investigators. J Am Coll Cardiol. 1997;29:498-505.

7. Nyman I, Areskog M, Areskog NH, Swahn E, Wallentin L. Very early risk stratification by electrocardiogram at rest in men with suspected unstable coronary heart disease. The RISC Study Group. J Intern Med. 1993;234:293-301.

8. Bertrand ME, Simoons ML, Fox KAA, Wallentin LC, Hamm CW, McFadden E, et al. Management of acute coronary syndromes in patients presenting without persistent ST-segment elevation. The Task Force on the management of acute coronary syndromes of the European Society of Cardiology. Eur Heart J. 2002;23:1809-40.

9. Arntz HR, Bossaert L, Filippatos GS. European Resuscitation Council Guidelines for Resuscitation 2005. Section 5. Initial management of acute coronary syndromes. Resuscitation. 2005; 67:S87-S96.

10. Álvarez Bueno M, Vera Almazán A, Rodríguez García JJ, Ferriz Martín JA, García Paredes T, García Alcántara A y Grupo del Proyecto ARIAM. Concepto, desarrollo y objetivos. Med Intensiva. 1999;23:273-9.

11. De Muyler R, Tonglet R, Nackers F, Boland B. Randomised evaluation of a specific training of general practitioners in cardiovascular prevention. Acta Cardiol. 2005;60:199-205. 